\author{
Junfang Xian \\ Zhengyu Zhang \\ Zhenchang Wang \\ Jing Li \\ Bentao Yang \\ Fengyuan Man \\ Qinglin Chang \\ Yunting Zhang
}

\section{Value of MR imaging in the differentiation of benign and malignant orbital tumors in adults}

Received: 24 September 2009

Revised: 24 November 2009

Accepted: 28 November 2009

Published online: 4 February 2010

(C) The Author(s) 2010.

This article is published with open access at Springerlink.com

J. Xian $(\bowtie) \cdot$ Z. Zhang $\cdot$ Z. Wang · J. Li · B. Yang · F. Man - Q. Chang Department of Radiology, Capital Medical University, Beijing Tongren Hospital, Beijing, 100730, China e-mail: cjr.xianjunfang@vip.163.com Tel.: +86-10-85111724

\section{Y. Zhang}

Department of Radiology, Tianjin

Medical University General Hospital, Tianjin, 300052, China

\begin{abstract}
Purpose: To prospectively evaluate magnetic resonance (MR) imaging including dynamic contrastenhanced MR imaging in the differentiation of benign from malignant orbital masses and to evaluate which MR imaging features are most predictive of malignant tumors. Materials and methods: The study was approved by the institutional review board and signed informed consent was obtained. Nonenhanced, static, and dynamic contrast-enhanced MR imaging was performed in 102 adult patients with an orbital mass. Diagnosis was based on histologic findings. MR imaging features of benign and malignant orbital lesions were evaluated correlated with histological findings. Multivariate logistic regression analysis was employed to identify the best combination
\end{abstract}

of MR imaging features that might be predictive of malignancy. Results: Nonenhanced, static, and dynamic enhancement MR imaging was significantly superior to two other models in prediction of malignancy $(p<0.05)$. Multivariate logistic regression analysis identified that the most discriminating MR imaging features were isointense mass on T2-weighted imaging and a washout-type time-intensity curve for both observers. Conclusion: Nonenhanced, static, and dynamic enhancement MR imaging improved differentiation between benign and malignant orbital masses in adult patients.

Keywords Orbit · Neoplasm · Magnetic resonance imaging . Contrast enhancement - Dynamic

\section{Introduction}

Computed tomography (CT) and magnetic resonance (MR) imaging are making an important impact on the characterization of orbital tumors and play an essential role in the management of these tumors [1-8]. It is well known that thoughtful analyses of CT and MR imaging of the orbit, along with careful history, clinical examination, and observation, are critical elements in the diagnosis and differential diagnosis of orbital tumors. In general, bony destruction of orbital wall on CT suggests malignant tumor, and hyperostosis underlies benign processes [1, 2, 8-11]. Tumors of the optic nerve sheath complex demonstrate characteristic features on $\mathrm{CT}$ and MR imaging contributing to the diagnosis and differential diagnosis [12-14]. Hyperintensity on T2-weighted imaging, and progressive and total homogeneous filling up of an orbital mass on dynamic contrast-enhanced MR imaging are considered as a pathognomonic sign of orbital cavernous hemangioma [1, $3,6,15-17]$. Marked enlargement of the orbital mass after compression of the neck on MR images is suggestive of venous varix $[1,6,16]$. Orbital cyst is characterized by no enhancement after contrast administration [4, 5, 8]. Orbital invasion from adjacent structures such as the paranasal sinus, globe, skull base, and nasopharynx shows soft tissue mass extended from these regions, and can be readily 
diagnosed by using imaging studies and easily confirmed by excisional biopsy $[8,18]$. These features can facilitate the differentiation of benign from malignant orbital soft tissue masses. Thus, these lesions are not the focus of our study.

However, the characterization of orbital lesions without the above-mentioned characteristic imaging findings as benign or malignant on the basis of CT and MR imaging features remains a challenge. It is still difficult to differentiate between benign and malignant diseases for these patients. Dynamic contrast-enhanced (DCE) MR imaging, a method that allows imaging of the physiology of the microcirculation, has been applied to the study of tumors of the breast, bone, bladder, prostate, head and neck, and other regions [19-22]. DCE MR imaging has also been applied to orbital tumors [17]. Nonetheless, little detailed information about the sensitivity and specificity of MR imaging including DCE MR imaging in the differentiation of benign from malignant orbital tumors has been reported. Our purpose was to prospectively evaluate static and DCE MR imaging combined with nonenhanced MR imaging in the differentiation of benign from malignant orbital masses that were difficult to differentiate on CT or conventional MR imaging, and to evaluate which MR imaging features are most predictive of orbital malignant tumors with associated interobserver variability.

\section{Materials and methods}

Patient population

Between June 2004 and February 2009, a total of 524 adult patients with a suspicious orbital soft tissue mass on CT were enrolled in the protocol, and MR imaging was performed in 502 patients. Two patients did not undergo MR imaging because of claustrophobia. Contrast-enhanced MR imaging was not performed in 20 patients who declined. Pathological data based on histological findings in surgical specimens were available for 309 patients. Two hundred and seven patients in line with exclusion criteria comprised 26 patients who had a history of surgery or treatment in the affected orbit, 6 patients whose images were poor, 47 patients with orbital invasion by lesions of adjacent structures such as the paranasal sinus, globe, skull base, and nasopharynx, 12 patients with optic nerve lesions, 23 patients who had orbital soft tissue masses with significant bony destruction or hyperostosis or lesions of the orbital wall on CT, 43 patients who showed hyperintensity on T2-weighted imaging, and progressive and total homogeneous filling up of an orbital mass on dynamic gadolinium-enhanced MR imaging, 16 patients with marked enlargement of the mass after compression of the neck on MR images, and 34 patients with no enhancement on contrast-enhanced MR imaging. Finally, 102 adult patients were eligible for participation.
Informed consent was obtained from all patients for performance of radiological studies and analysis of clinical data anonymously. Our institutional review board approved the study protocol.

\section{MR imaging}

MR imaging was performed on a General Electric (GE) Signa 1.5-T MR imaging system (GE Healthcare, Milwaukee, WI) with an 8-channel head coil.

MR imaging was performed with T1-weighted (repetition time /echo time, 400/15 ms) and T2-weighted $(3,000 / 120)$ fast spin-echo images. A $16-\mathrm{cm}$ field of view, a 3 -mm-thick section at 0 interval, and a $288 \times 256$ matrix with two signals acquired were used. DCE MR imaging was performed by using a fast spoiled gradient recalled (FSPGR) sequence with $8.4 / 4 \mathrm{~ms}$, a flip angle of $15^{\circ}$, one excitation, a matrix of $256 \times 160$, a field of view of $220 \times 220 \mathrm{~mm}$, and a section thickness of $3.2 \mathrm{~mm}$ at 0 interval. A power injector (Medrad, Indianola, Pa) with an injection flow rate of $2 \mathrm{~mL} / \mathrm{s}$ was used. The acquisition of the dynamic images began concurrently with the initiation of the injection of $0.1 \mathrm{mmol}$ of gadopentetate dimeglumine (Magnevist, Schering, Berlin, Germany) per kilogram. The contrast material injection was followed by a $20-\mathrm{mL}$ saline flush. In DCE MR imaging scanning, a total of 12 scans were obtained. For each scan, 12 sections were obtained at $13 \mathrm{~s}$. The interval between two scans was $12 \mathrm{~s}$. Total scanning time of DCE MR imaging was $288 \mathrm{~s}$ (4 min $48 \mathrm{~s}$ ).

\section{Image interpretation}

DCE MR imaging was evaluated using a GE AW 4.2 workstation (GE Healthcare, Milwaukee, Wisconsin). A region of interest (ROI) was drawn manually on the dynamic images for assessment of the enhancement kinetics. Observers were instructed to choose the area that demonstrated the greatest degree of early enhancement so that time-intensity curves (TICs) could be generated. The ROI area was typically $8 \mathrm{~mm}^{2}$. The contrast index (CI) was calculated from the following equation: $\mathrm{CI}=[$ signal intensity (post-contrast) - signal intensity (pre-contrast)]/ signal intensity (pre-contrast). Tpeak was the time to peak enhancement. The TICs were analyzed qualitatively as washout, persistent, or plateau-shaped curves.

Two experienced head and neck radiologists (15 years of experience; 8 years of experience), blinded to the diagnosis, clinical history, and results of other imaging modalities, independently evaluated MR imaging features on MR imaging including DCE MR imaging. Each observer evaluated MR imaging features and diagnosed the lesion as benign or malignant with a five-point confidence rating ( 1 , definitely benign; 2 , uncertain benign; 3 , undetermined; 4 , uncertain malignant; and 5 , definitely malignant). 
Observers were trained in MR image interpretation before the investigation was initiated.

\section{Statistical analysis}

Univariate logistic regression models were fit for each feature in an attempt to characterize the predictive ability of each feature for identifying a malignant lesion.

The frequency distribution of individual MR imaging features in the benign group was compared with that in the malignant group by using chi-square tests. The Fisher exact test was performed when the sample size in the subgroups was deemed too small. A $P$ value of less than 0.05 was considered to represent a significant difference. Interobserver agreement of individual MR imaging features was determined by means of $\kappa$ analysis. A $\kappa$ value of less than 0.40 indicated poor agreement; that equal to or greater than 0.40 and less than 0.60 , moderate agreement; that equal to or greater than 0.60 and less than 0.80 , good agreement; and that equal to or greater than 0.80 , excellent agreement.

Multivariate logistic regression analysis was employed to identify the most important MR imaging features predictive of orbital malignancy. Nonenhanced MR imaging features (model 1), combination of nonenhanced and static contrast-enhanced MR imaging features (model 2), and DCE MR imaging in combination with nonenhanced and static contrast-enhanced MR imaging features (model 3 ) were evaluated respectively. Final selection of multivariate predictors (model 4) was determined with stepwise analysis as a backward-stepping procedure based on a likelihood ratio test with a $P$ value greater than 0.10 used for exclusion from the model. The regression coefficient, $b$, of the selected variables of model 4 provided an estimate of the extent to which each MR imaging feature contributed to the diagnostic accuracy. The Hosmer-Lemeshow goodness-of-fit test was used to assess model fit.

Results of subjective MR imaging diagnosis were evaluated for sensitivity and specificity with a confidence rating of uncertain benign, undetermined, uncertain malignant, and definitely malignant as positive results requiring histologic biopsy or surgery and with a confidence rating of definitely benign as a negative result.

Data analysis was performed with statistical software (SPSS for Windows, version 10.0; Chicago, Ill).

\section{Results}

\section{Diagnosis}

Orbital soft tissue masses were histologically confirmed in all 102 patients (51 male and 51 female; mean age 50 years, range 18-89 years). Benign lesions were demonstrated in 60 patients (22 male and 38 female; mean age 48 years, range 20-76 years), and malignant lesions in 42 patients (29 male and 13 female; mean age 52 years, range 18 89 years). There was significant difference in the sex of patients with benign versus malignant lesions $(p=0.001)$. There was no significant difference in the age of patients with benign versus malignant lesions $(p=0.205)$. The left orbit was involved in 24 patients with benign lesions and in 22 patients with malignant lesions. The right orbit was involved in 34 patients with benign lesions and in 16 patients with malignant lesions. Bilateral orbits were involved in 2 patients with benign lesions and in 4 patients with malignant lesions. There was no significant difference in the side involving the mass between benign versus malignant lesions ( $p=0.123$ ).

Sixty benign lesions comprised 22 pleomorphic adenomas of the lacrimal gland (21.6\%), 16 schwannomas $(15.7 \%), 15$ inflammatory pseudotumors $(14.7 \%), 5$ lymphangiomas $(4.9 \%)$, and 2 solitary neurofibromas (2\%). Forty-two malignant lesions comprised 27 lymphomas $(26.5 \%), 6$ adenoid cystic carcinomas of the lacrimal gland $(5.9 \%), 5$ adenocarcinomas of the lacrimal gland (4.9\%), 2 metastases (2\%), 1 pleomorphic adenocarcinoma of the lacrimal gland (1\%), and 1 rhabdomyosarcoma (1\%).

Frequency distribution of individual MR imaging features and interobserver agreement between two observers

Tables 1 and 2 describe the frequency distribution of nonenhanced MR imaging features (Table 1) and static and DCE MR imaging features (Table 2), correlation of these features with pathological diagnosis (benign or malignant tumors) for observer 1, as well as interobserver agreement between observers 1 and 2. For both observers, there was significant difference between the benign group and the malignant group in the location, shape, and margin of the mass, homogeneity on T1- and T2-weighted imaging, signal intensity on T2-weighted imaging, pattern of enhancement, and type of time-intensity curve (TIC) $(P$ $<0.05$ ) (Figs. 1, 2, 3, and 4). For both observers, tumor location including extraconal space and anterior orbit preseptal space, irregular shape, ill-defined margin, molding around orbital structures, a homogeneous mass on T1- and T2-weighted imaging, isointensity relative to extraocular muscle on T2-weighted imaging, homogeneous enhancement, and washout-type TIC were correlated $(P<0.05)$ with the diagnosis of malignancy (Figs. 1, 2, and 3 ).

Tumor location, shape, margin, homogeneity on T1- and T2-weighted imaging, signal intensity on T2-weighted imaging, molding around orbital structures, globe indentation caused by the tumor, pattern of enhancement, and washout-type TIC had good to excellent interobserver agreement. Other MR imaging features had moderate to poor interobserver agreement. 
Table 1 Frequency distribution and correlation with final diagnosis of nonenhanced MR imaging parameters for observer 1 and interobserver agreement

\begin{tabular}{|c|c|c|c|c|c|c|c|c|}
\hline \multirow[t]{3}{*}{ MR imaging feature } & \multicolumn{6}{|c|}{ Type of disease } & \multirow[t]{3}{*}{$P$ value } & \multirow[t]{3}{*}{$\kappa$ value $^{\mathrm{b}}$} \\
\hline & \multicolumn{2}{|c|}{ Overall } & \multicolumn{2}{|c|}{ Benign } & \multicolumn{2}{|c|}{ Malignant } & & \\
\hline & $N$ & $\%$ & $N$ & $\%$ & $N$ & $\%$ & & \\
\hline No. of patients & 102 & & 60 & & 42 & & & \\
\hline \multicolumn{9}{|l|}{ Tumor location } \\
\hline Intraconal & 48 & 47 & 25 & 42 & 23 & 55 & 0.192 & 0.902 \\
\hline Extraconal & 58 & 57 & 27 & 45 & 31 & 74 & 0.004 & 0.824 \\
\hline Lacrimal fossa & 50 & 49 & 29 & 48 & 21 & 50 & 0.868 & 0.625 \\
\hline Anterior orbit preseptal & 42 & 41 & 14 & 23 & 28 & 67 & $<0.001$ & 0.647 \\
\hline No. of compartments involved & & & & & & & $<0.001$ & 0.509 \\
\hline One compartment & 46 & 45 & 38 & 63 & 8 & 19 & & \\
\hline Two compartments & 26 & 25 & 12 & 20 & 14 & 33 & & \\
\hline Three compartments & 19 & 19 & 7 & 12 & 12 & 29 & & \\
\hline Four compartments & 11 & 11 & 3 & 5 & 8 & 19 & & \\
\hline No. of masses & & & & & & & 0.026 & 0.496 \\
\hline Solitary & 75 & 74 & 49 & 82 & 26 & 62 & & \\
\hline Multiple & 27 & 26 & 11 & 18 & 16 & 38 & & \\
\hline Shape & & & & & & & $<0.001$ & 0.802 \\
\hline Regular & 42 & 41 & 34 & 57 & 8 & 19 & & \\
\hline Irregular & 60 & 59 & 26 & 43 & 34 & 81 & & \\
\hline Margin & & & & & & & $<0.001$ & 0.649 \\
\hline Well defined & 62 & 61 & 48 & 80 & 14 & 33 & & \\
\hline Ill defined & 40 & 39 & 12 & 20 & 28 & 67 & & \\
\hline \multicolumn{9}{|c|}{ Relationship between masses and adjacent structures } \\
\hline Molding structures & 53 & 52 & 18 & 30 & 35 & 83 & $<0.001$ & 0.727 \\
\hline Displacement & 92 & 90 & 56 & 93 & 36 & 86 & 0.203 & 0.24 \\
\hline Globe indentation & 25 & 25 & 18 & 30 & 7 & 17 & 0.123 & 0.678 \\
\hline Extraorbital involvement & 21 & 21 & 9 & 15 & 12 & 29 & 0.095 & 0.468 \\
\hline T1 signal intensity ${ }^{a}$ & & & & & & & 0.003 & 0.271 \\
\hline Hypointense & 15 & 15 & 14 & 23 & 1 & 2 & & \\
\hline Isointense & 87 & 85 & 46 & 77 & 41 & 98 & & \\
\hline Hyperintense & 0 & 0 & 0 & 0 & 0 & 0 & & \\
\hline T1 homogeneity & & & & & & & 0.018 & 0.622 \\
\hline Homogeneous & 72 & 71 & 37 & 62 & 35 & 83 & & \\
\hline Inhomogeneous & 30 & 29 & 23 & 38 & 7 & 17 & & \\
\hline $\mathrm{T} 2$ signal intensity $^{\mathrm{a}}$ & & & & & & & $<0.001$ & 0.694 \\
\hline Hypointense & 7 & 7 & 6 & 10 & 1 & 2 & & \\
\hline Isointense & 56 & 55 & 21 & 35 & 35 & 84 & & \\
\hline Hyperintense & 39 & 38 & 33 & 55 & 6 & 14 & & \\
\hline T2 homogeneity & & & & & & & $<0.001$ & 0.624 \\
\hline Homogeneous & 54 & 53 & 23 & 38 & 31 & 74 & & \\
\hline Inhomogeneous & 48 & 47 & 37 & 62 & 11 & 26 & & \\
\hline
\end{tabular}

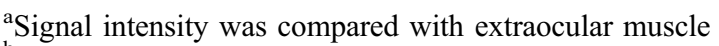

${ }^{\mathrm{b}} \mathrm{A} \kappa$ value (interobserver agreement) less than 0.40 represented poor agreement; that equal to or greater than 0.40 and less than 0.60 , moderate agreement; that equal to or greater than 0.60 and less than 0.80 , good agreement; and that equal to or greater than 0.80 , excellent agreement

Involvement of preseptal space, involvement of one compartment, margin, molding around orbital structures, an isointense mass relative to extraocular muscle on T1-and T2-weighted imaging, a homogeneous mass on T2-weighted imaging, homogeneous enhancement, and washout-type TIC had a high sensitivity and specificity for diagnosing malignant versus benign processes (Table 3). Other MR imaging features had a lower sensitivity or specificity (Table 3 ). 
Table 2 Frequency distribution and correlation with final diagnosis of static and dynamic contrast-enhanced MR imaging features for observer 1 and interobserver agreement

\begin{tabular}{|c|c|c|c|c|c|c|c|c|}
\hline \multirow[t]{3}{*}{ MR imaging feature } & \multicolumn{6}{|c|}{ Type of disease } & \multirow[t]{3}{*}{$P$ value } & \multirow[t]{3}{*}{$\kappa$ value $^{\mathrm{a}}$} \\
\hline & \multicolumn{2}{|c|}{ Overall } & \multicolumn{2}{|c|}{ Benign } & \multicolumn{2}{|c|}{ Malignant } & & \\
\hline & $N$ & $\%$ & $N$ & $\%$ & $N$ & $\%$ & & \\
\hline No. of patients & 102 & & 60 & & 42 & & & \\
\hline \multicolumn{9}{|l|}{ Static enhancement } \\
\hline Pattern of enhancement & & & & & & & $<0.001$ & 0.628 \\
\hline Homogeneous & 49 & 48 & 20 & 33 & 29 & 69 & & \\
\hline Inhomogeneous & 50 & 49 & 37 & 62 & 13 & 31 & & \\
\hline Peripheral & 3 & 3 & 3 & 5 & 0 & 0 & & \\
\hline \multicolumn{9}{|l|}{ Dynamic enhancement } \\
\hline CI value & & & & & & & 0.476 & 0.457 \\
\hline $\mathrm{CI} \leq 0.5$ & 5 & 5 & 3 & 5 & 2 & 5 & & \\
\hline $0.5<\mathrm{CI} \leq 1$ & 53 & 52 & 30 & 50 & 23 & 54 & & \\
\hline $1<\mathrm{CI} \leq 1.5$ & 27 & 26 & 14 & 23 & 13 & 31 & & \\
\hline $1.5<\mathrm{CI} \leq 2$ & 15 & 15 & 13 & 22 & 2 & 5 & & \\
\hline $\mathrm{CI}>2$ & 2 & 2 & 0 & 0 & 2 & 5 & & \\
\hline Tpeak (ms) & & & & & & & 0.200 & 0.576 \\
\hline $\mathrm{T} \leq 50$ & 7 & 7 & 3 & 5 & 4 & 10 & & \\
\hline $50<\mathrm{T} \leq 75$ & 90 & 88 & 53 & 88 & 37 & 88 & & \\
\hline $75<\mathrm{T} \leq 100$ & 3 & 3 & 2 & 3 & 1 & 2 & & \\
\hline $100<\mathrm{T} \leq 125$ & 2 & 2 & 2 & 3 & 0 & 0 & & \\
\hline $\mathrm{T}>125$ & 0 & 0 & 0 & 0 & 0 & 0 & & \\
\hline TIC type & & & & & & & $<0.001$ & \\
\hline Persistent & 28 & 27 & 28 & 47 & 0 & 0 & & 0.535 \\
\hline Plateau-shaped & 25 & 25 & 18 & 30 & 7 & 17 & & 0.469 \\
\hline Washout & 49 & 48 & 14 & 23 & 35 & 83 & & 0.843 \\
\hline
\end{tabular}

${ }^{\mathrm{a}} \mathrm{A} \kappa$ value (interobserver agreement) less than 0.40 represented poor agreement; that equal to or greater than 0.40 and less than 0.60 , moderate agreement; that equal to or greater than 0.60 and less than 0.80 , good agreement; and that equal to or greater than 0.80 , excellent agreement

\section{Combination of orbital MR imaging features}

Results of observers 1 and 2 are delineated in Table 4. Logistic regression model 3 , based on the combination of nonenhanced, static, and dynamic contrast-enhanced MR imaging features, had a higher ability to predict malignancy than did logistic regression models 1 and $2(p<0.05)$ with a sensitivity of $83.3 \%$ and $83.3 \%$ and a specificity of $88.3 \%$ and $91.7 \%$ for observers 1 and 2, respectively (Table 4). Stepwise multivariate logistic regression analysis (model 4) with all evaluated MR imaging features revealed that only two MR imaging features were significant predictors of malignancy for observer 1 and three MR imaging features were significant predictors of malignancy for observer 2 (Table 4). The discriminating MR imaging features between benign and malignant orbital masses of observer 1 were isointense mass relative to extraocular muscle on T2-weighted imaging $(b=1.452,95 \%$ confidence interval 1.359-13.417) and a washout-type TIC $(b=1.452,95 \%$ confidence interval $1.359-13.417)$. The discriminating MR imaging features of observer 2 were involvement of preseptal space $(b=1.637,95 \%$ confidence interval 1.458-18.104), isointense mass relative to extraocular muscle on T2-weighted imaging $(b=1.512,95 \%$ confidence interval 1.379-14.930), and a washout-type TIC $(b=2.3,95 \%$ confidence interval 3.034-32.081).

The Hosmer-Lemeshow goodness-of-fit test results were not significantly different for each model and indicated that all the models were adequately fitted.

\section{Confidence of subjective MR imaging diagnosis}

According to our five-point confidence rating scale of uncertain benign, undetermined, uncertain malignant, or definitely malignant as positive results requiring histological biopsy or surgery and with a confidence rating of definitely benign as a negative result, sensitivity of nonenhanced MR imaging features was $81 \%(34 / 42)$ and $81 \%(34 / 42)$ and specificity was $58.3 \%(35 / 60)$ and $68.3 \%$ $(41 / 60)$ for observers 1 and 2, respectively. Sensitivity and specificity of nonenhanced combined with static contrast- 

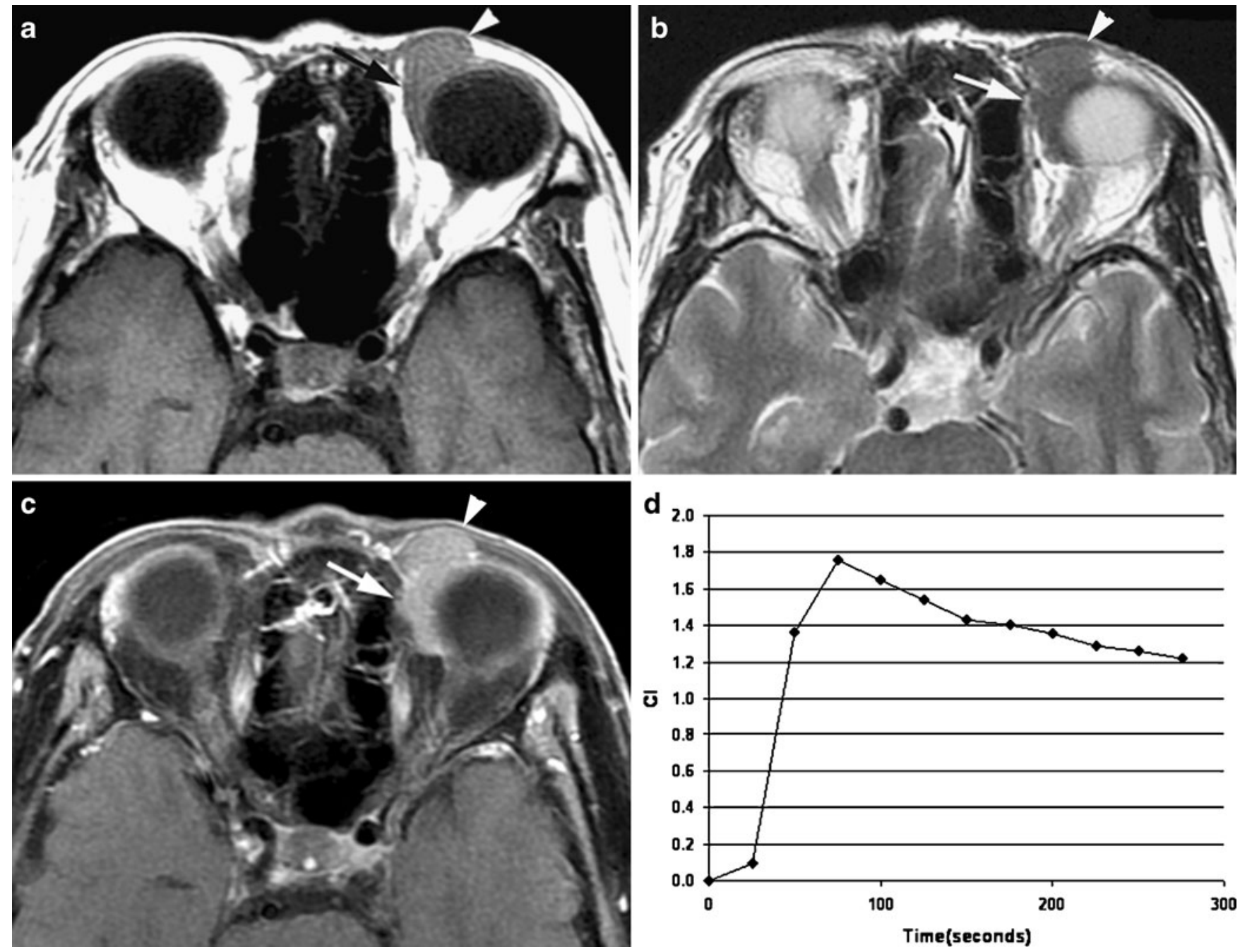

Fig. 1 Transverse MR images in a 79-year-old man demonstrated a well-circumscribed mass molding around the globe in the left preseptal space (arrowhead) and extraconal space (arrow). a T1weighted image (400/15) showed a homogeneously isointense mass relative to extraocular muscle. b T2-weighted image $(3,000 / 120)$ showed a homogeneously isointense mass relative to extraocular

enhanced MR imaging features were the same as nonenhanced MR imaging features for observers 1 and 2 . Sensitivity of nonenhanced combined with static and dynamic contrast-enhanced MR imaging features was $97.6 \%(41 / 42)$ and $100 \%(42 / 42)$ and specificity was $71.7 \%(43 / 60)$ and $73.3 \%(44 / 60)$ for observers 1 and 2 , respectively, significantly higher than those of other two models $(p<0.05)$.

\section{Discussion}

Ben Simon et al. reported that none of the orbital imaging features, including CT and MR imaging features, had a

muscle. c Static contrast-enhanced MR image (400/15) with fat suppression showed mildly homogeneous enhancement of the mass. d Time-intensity curve for this patient was characterized as a washout curve. Pathologic examination revealed a non-Hodgkin's lymphoma

high enough sensitivity to distinguish between malignant and benign orbital tumors [8]. However, our study has shown that some MR imaging features are associated with a malignant disease and have good to excellent interobserver agreement between two observers. Of these features, involvement of preseptal space, ill-defined margin, molding around orbital structures, isointensity on T2weighted imaging, a homogeneous mass on T2-weighted imaging, homogeneous enhancement, and washout-type TIC had a high sensitivity and specificity for diagnosing malignant disease. Multivariate logistic regression analysis revealed that the most predictive features for malignancy were isointense mass on T2-weighted imaging and a washout-type TIC for both observers. 

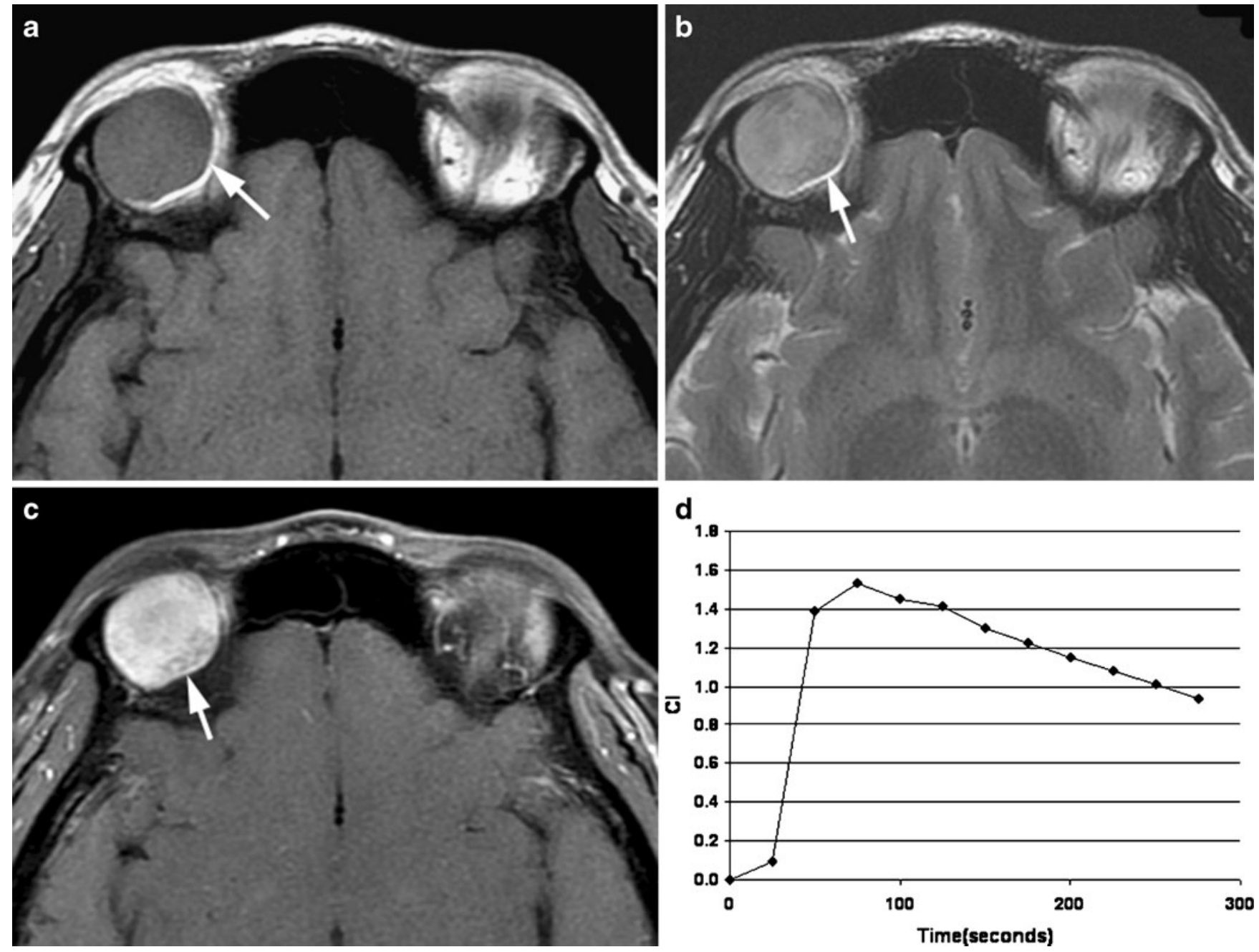

Fig. 2 Transverse MR images in a 30-year-old man identified an oval well-defined mass (arrow) in the right lacrimal gland. a T1weighted image (400/15) showed a homogeneously isointense mass relative to extraocular muscle. b T2-weighted image $(3,000 / 120)$ showed an inhomogeneously hyperintense mass relative to extraoc- ular muscle. c Static contrast-enhanced MR image (400/15) with fat suppression showed inhomogeneous enhancement of the mass. d Time-intensity curve for this patient was characterized as a washout curve. Pathologic examination revealed an adenoid cystic carcinoma of the lacrimal gland
Orbital lymphoma, the most common primary orbital malignancy in adults, usually manifests as a diffuse, solid, enhancing mass with molding around the globe on imaging studies, reflecting the irregular infiltration of orbital structures [23-27]. Our results revealed that an irregular mass was seen in all 27 patients with lympho$\mathrm{ma}$, in which soft tissue mass was ill defined in 22 patients $(81.5 \%)$ and well defined in only five patients $(18.5 \%)$. However, a circumscribed round or oblong mass, seen in $48 \%$ to $54 \%$ of the patients with orbital lymphoma on MRI or CT reported in the literature [25, 26], does not rule out orbital lymphoma. Our results and recent literature $[25,26]$ indicated that orbital lymphoma appeared to be isointense compared with extraocular muscles on T1-weighted and T2-weighted images. This was contrary to earlier studies that showed orbital lymphoma appeared to be isointense compared with extraocular muscles on T1-weighted images and hyperintense on T2-weighted imaging [27], but this may explain one of the most predictive features for malignancy-isointense mass on T2-weighted imaging.

Primary epithelial malignancies of the lacrimal gland are the second most common malignant tumors in the orbit [23, 24]. Generally, a small circumscribed lesion in the lacrimal gland is more likely to be benign and a larger or poorly circumscribed tumor is more likely to be malignant on CT or MR imaging $[5,10,11,28]$. Erosion of bone can occur, even when the primary epithelial malignant tumor is still 

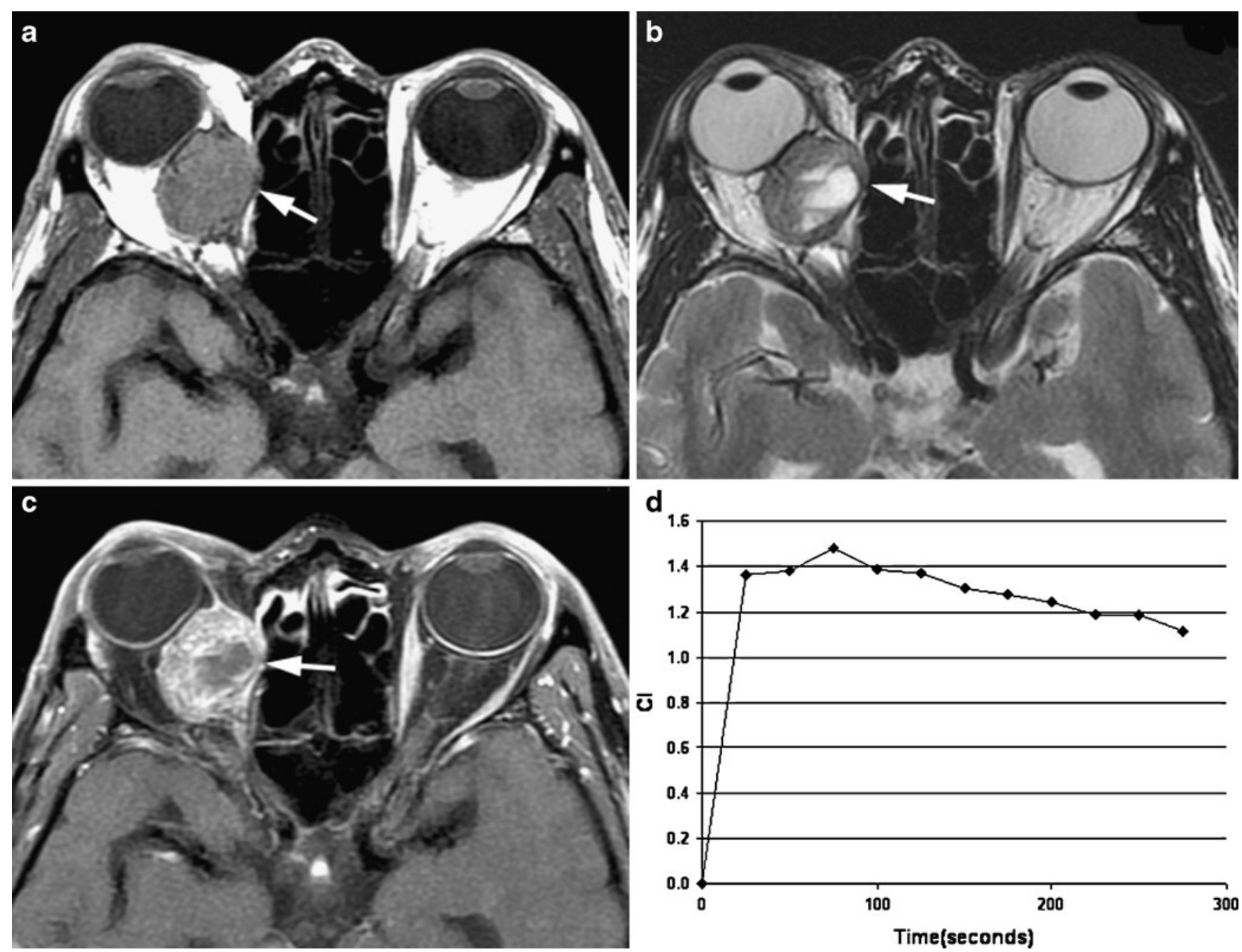

Fig. 3 Transverse MR images in a 49-year-old man revealed an oval well-defined mass (arrow) in right medial rectus muscle. a T1weighted image $(400 / 15)$ showed a homogeneously isointense mass relative to extraocular muscle. b T2-weighted image $(3,000 / 120)$ showed an inhomogeneously isointense mass relative to extraocular

muscle with patchy hyperintensity within the mass. c Static contrastenhanced MR image (400/15) with fat suppression showed inhomogeneous enhancement of the mass. d Time-intensity curve for this patient was characterized as a washout curve. Pathologic examination revealed a metastasis from lung adenocarcinoma

small [28]. However, primary epithelial malignancies of the lacrimal gland may demonstrate a round or ovoid welldefined soft tissue mass without bony erosion [28], which was seen in four patients with adenoid cystic carcinoma of the lacrimal gland in our study. For these patients, shape or margin of the mass cannot be used to distinguish them from benign disease, but three of these four patients with orbital lymphoma showed washout-type TICs which were suggestive of malignancy. Because bony destruction of orbital wall depicted on CT suggested malignant tumor, malignant tumors of the lacrimal gland with bony destruction were not included in our study.

In clinical practice, some malignant orbital tumors are frequently misinterpreted as benign on nonenhanced MR

imaging or static enhanced MR imaging, perhaps because of their small size, well-defined margins, and slow progression [8]. However, these malignant orbital tumors will demonstrate washout-type TICs on dynamic contrastenhanced MR imaging, which may help flag up benignappearing lesions and allow less experienced radiologists to target lesions that need further work-up in a referral center.

Compared with the surface coil, the resolution of the 8channel head coil is limited. However, the orbital apex is poorly demonstrated on images obtained with the surface coil. Thus, we chose the 8-channel head coil rather than the surface coil.

Limitations of this study included patient population bias and the small sample size. Tumors included in the 

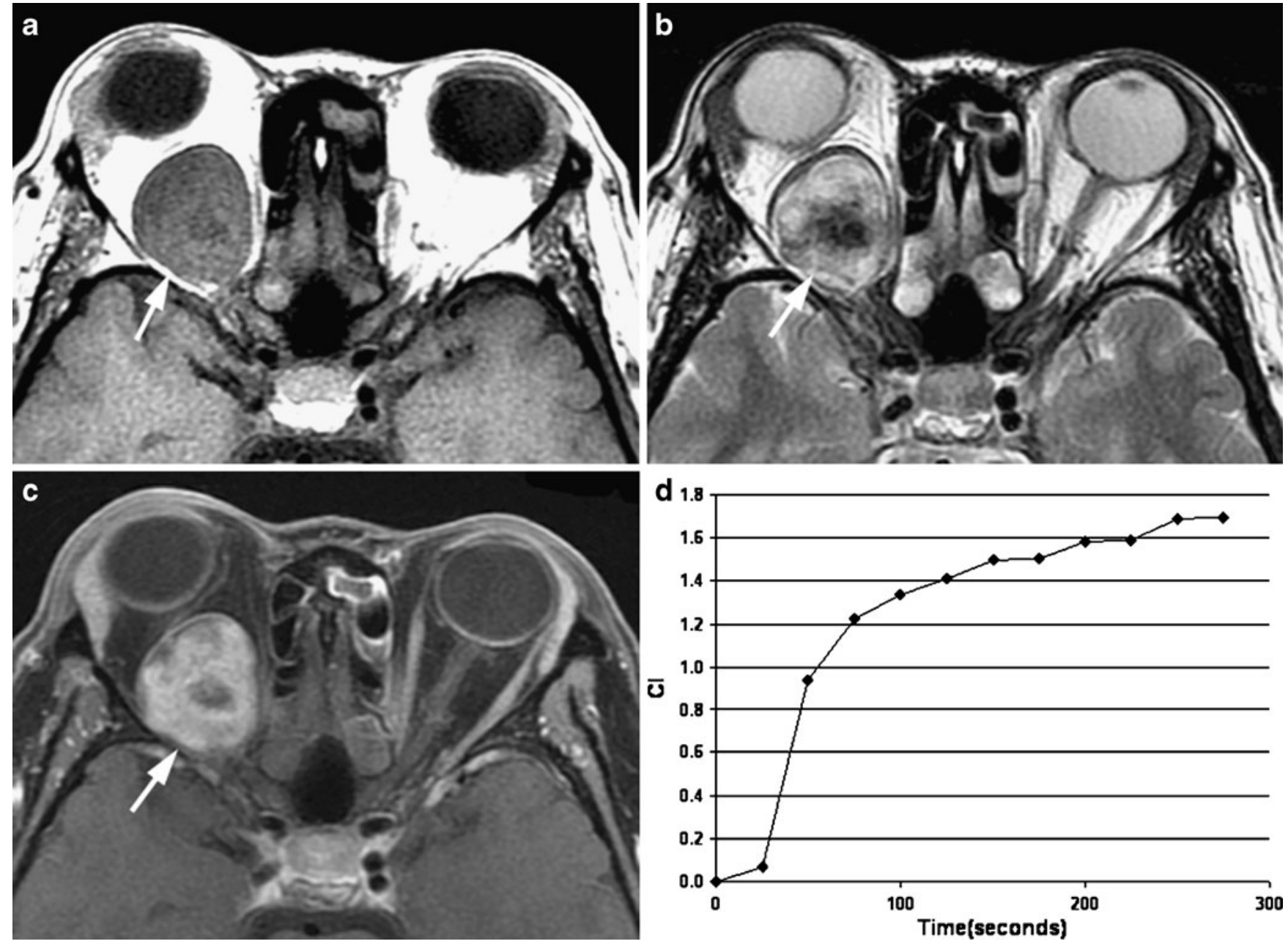

Fig. 4 Transverse MR images in a 45-year-old woman displayed an oval well-defined mass (arrow) in right intraconal space. a T1weighted image (400/15) showed an inhomogeneously isointense mass relative to extraocular muscle. b T2-weighted image $(3,000 / 120)$ showed an inhomogeneously hyperintense mass relative

study reflect those that are more difficult to differentiate benign from malignant by using imaging studies and the referral pattern to our orbital clinic. Tumors that are easier to distinguish benign from malignant by using imaging studies are not included in our study, in which more benign lesions were excluded. In addition, the analysis of dynamic enhanced MR imaging was limited to qualitative analysis and simple descriptive statistics and aimed to evaluate predominant clinical practices. Advanced modeling will be investigated in future work.

In conclusion, the most predictive features for malignancy were isointense mass on T2-weighted imaging and a washout-type TIC. In addition, location, shape, and margin of the mass, molding around orbital structures, and pattern of enhancement were critical features of orbital masses that should be considered in lesion characterization. The combination of nonenhanced, static,

to extraocular muscle. c Static contrast-enhanced MR image $(400 / 15)$ with fat suppression showed inhomogeneous enhancement of the mass. d Time-intensity curve for this patient was characterized as a persistent curve. Pathologic examination revealed a schwannoma

and dynamic contrast-enhanced MR imaging was superior to combination of nonenhanced and static contrastenhanced MR imaging and nonenhanced MR imaging alone in the differentiation of benign from malignant soft tissue lesions in the orbit. The addition of dynamic contrast-enhanced MR imaging substantially enhances the accuracy of MR imaging in the differentiation of benign from malignant orbital masses and facilitates prediction of malignancy. Advances in the techniques of MR imaging and the ability to interpret orbital MR imaging will optimize the decision-making process in treatment planning, determining the most efficient and least invasive pathways toward helping our patients with their disease. The multivariate models can serve an important role as a training and decision support system for assisting less experienced radiologists practicing orbital MR imaging. 
Table 3 Positive predictive values, negative predictive values, sensitivity, and specificity of MR imaging features in evaluating malignant versus benign diseases in patients with orbital soft tissue masses

\begin{tabular}{|c|c|c|c|c|c|c|c|c|}
\hline & \multicolumn{2}{|c|}{ Positive predictive value $^{\mathrm{a}}$} & \multicolumn{2}{|c|}{ Negative predictive value ${ }^{b}$} & \multicolumn{2}{|c|}{ Sensitivity $^{\mathrm{c}}$} & \multicolumn{2}{|c|}{ Specificity $^{\mathrm{d}}$} \\
\hline & $n$ & $\%$ & $n$ & $\%$ & $n$ & $\%$ & $n$ & $\%$ \\
\hline No. of patients & & & & & 42 & & 60 & \\
\hline Solitary mass & 49 & 65.3 & 16 & 59.3 & 49 & 81.7 & 16 & 38.1 \\
\hline Extraconal involvement & 31 & 53.4 & 33 & 75.0 & 31 & 73.8 & 33 & 55 \\
\hline Preseptal involvement & 28 & 66.7 & 46 & 76.7 & 28 & 66.7 & 46 & 76.7 \\
\hline Shape & 34 & 56.7 & 34 & 81.0 & 34 & 81.0 & 34 & 56.7 \\
\hline Margin & 28 & 70.0 & 48 & 77.4 & 28 & 66.7 & 48 & 80.0 \\
\hline Molding structures & 35 & 66.0 & 42 & 85.7 & 35 & 83.3 & 42 & 70.0 \\
\hline Inhomogeneous T1 & 37 & 51.4 & 7 & 23.3 & 37 & 61.7 & 7 & 16.7 \\
\hline Homogeneous T1 & 35 & 48.6 & 23 & 76.7 & 35 & 83.3 & 23 & 38.3 \\
\hline T1 hypointense & 1 & 6.7 & 46 & 52.9 & 1 & 2.4 & 46 & 76.7 \\
\hline $\mathrm{T} 1$ isointense & 41 & 47.1 & 14 & 93.3 & 41 & 97.6 & 46 & 76.7 \\
\hline Inhomogeneous $\mathrm{T} 2$ & 12 & 22.6 & 19 & 38.8 & 12 & 28.6 & 19 & 31.7 \\
\hline Homogeneous T2 & 30 & 61.2 & 41 & 77.4 & 30 & 71.4 & 41 & 68.3 \\
\hline $\mathrm{T} 2$ hypointense & 1 & 14.3 & 54 & 56.8 & 1 & 2.4 & 54 & 90.0 \\
\hline $\mathrm{T} 2$ isointense & 35 & 62.5 & 39 & 84.8 & 35 & 83.3 & 39 & 65.0 \\
\hline T2 hyperintense & 6 & 15.4 & 27 & 42.9 & 6 & 14.3 & 27 & 45.0 \\
\hline Homogeneous enhancement & 29 & 59.2 & 40 & 75.5 & 29 & 69.0 & 40 & 66.7 \\
\hline Inhomogeneous enhancement & 13 & 26.0 & 23 & 44.2 & 13 & 31.0 & 23 & 38.3 \\
\hline Peripheral enhancement & 0 & 0.0 & 57 & 57.6 & 0 & 0.0 & 57 & 95.0 \\
\hline Persistent-type TIC & 28 & 100 & 42 & 56.8 & 28 & 46.7 & 42 & 100 \\
\hline Washout-type TIC & 35 & 71.4 & 46 & 86.8 & 35 & 83.3 & 46 & 76.7 \\
\hline One compartment involved & 33 & 60.0 & 38 & 80.9 & 33 & 78.6 & 38 & 63.3 \\
\hline Two compartments involved & 20 & 66.7 & 50 & 69.4 & 20 & 47.6 & 50 & 83.3 \\
\hline Three compartments involved & 8 & 72.7 & 57 & 62.6 & 8 & 19.5 & 57 & 95.0 \\
\hline
\end{tabular}

Only features with significantly different occurrence between malignant and benign groups were included in the calculation

${ }^{a}$ Rate of detecting disease among patients with positive test results equals number of patients with disease (malignant) and positive test results per number of patients with positive test results

${ }^{\mathrm{b}}$ Rate of detecting nondisease among patients without positive test results equals number of patients without disease (malignant) and negative test results per number of patients with negative results

${ }^{\mathrm{c}}$ Rate of positive test results among patients with disease equals number of patients with disease (malignant) and positive test results per number of patients with disease (malignant)

${ }^{\mathrm{d}}$ Rate of negative test results among patients without disease equals number of patients without disease (malignant) and negative test results per number of patients without disease (malignant)

Table 4 Logistic regression models of MR imaging parameters for observers 1 and 2: analysis of 102 patients with orbital soft tissue mass

\begin{tabular}{|c|c|c|c|c|c|c|}
\hline \multirow[t]{2}{*}{ Model } & \multicolumn{2}{|c|}{$\begin{array}{l}\text { No. of MR imaging } \\
\text { features }\end{array}$} & \multicolumn{2}{|c|}{ Sensitivity $(n=42)$} & \multicolumn{2}{|c|}{ Specificity $(n=60)$} \\
\hline & $\begin{array}{l}\text { Observer } \\
1\end{array}$ & $\begin{array}{l}\text { Observer } \\
2\end{array}$ & $\begin{array}{l}\text { Observer } \\
1\end{array}$ & $\begin{array}{l}\text { Observer } \\
2\end{array}$ & $\begin{array}{l}\text { Observer } \\
1\end{array}$ & $\begin{array}{l}\text { Observer } \\
2\end{array}$ \\
\hline 1 Nonenhanced MR imaging features & 21 & 21 & $78.6(33)^{\mathrm{a}}$ & $76.2(32)$ & $86.7(52)$ & $83.3(50)$ \\
\hline 2 Nonenhanced combined with static CE MR imaging features & 23 & 23 & $78.6(33)$ & $73.8(31)$ & $88.3(53)$ & $86.7(52)$ \\
\hline $\begin{array}{l}3 \text { Nonenhanced combined with static and DCE MR imaging } \\
\text { features }\end{array}$ & 27 & 27 & $83.3(35)$ & $83.3(35)$ & $88.3(53)$ & $91.7(55)$ \\
\hline 4 Stepwise logistic regression analysis of model 3 & $2^{\mathrm{b}}$ & $3^{\mathrm{c}}$ & $69.0(29)$ & $78.6(33)$ & $83.3(50)$ & $85.0(51)$ \\
\hline
\end{tabular}

Differences between models 1 and 2, models 2 and 3, and models 1 and 3 were all significant $(p<0.05)$ for observer 1 . For observer 2 , differences between models 1 and 3, and models 2 and 3 were significant $(p<0.05)$, but difference between models 1 and 2 was insignificant $(p>0.05)$

${ }^{a}$ Numbers are percentages. Numbers in parentheses were used to calculate the percentages

${ }^{\mathrm{b}} \mathrm{MR}$ imaging features included isointensity on T2-weighted imaging and washout-type TIC

${ }^{\mathrm{c}} \mathrm{MR}$ imaging features included isointensity on T2-weighted imaging, washout-type TIC, and anterior orbit preseptal involvement 
Acknowledgements This work was in part supported by a grant (2004-B-31) from the Beijing Science and Technology Committee, China, and grants $(7062019,7082026)$ from Beijing Nature and Science, China
Open Access This article is distributed under the terms of the Creative Commons Attribution Noncommercial License which permits any noncommercial use, distribution, and reproduction in any medium, provided the original author(s) and source are credited.

\section{References}

1. Mafee MF, Putterman A, Valvassori GE, Campos M, Capek V (1987) Orbital space-occupying lesions: role of computed tomography and magnetic resonance imaging. An analysis of 145 cases. Radiol Clin North Am 25:529559

2. Warner MA, Weber AL, Jakobiec FA (1996) Benign and malignant tumors of the orbital cavity including the lacrimal gland. Neuroimaging Clin North Am 6:123-142

3. Ansari SA, Mafee MF (2005) Orbital cavernous hemangioma: role of imaging. Neuroimaging Clin North Am 15:137-158

4. Lemke AJ, Kazi I, Felix R (2006) Magnetic resonance imaging of orbital tumors. Eur Radiol 16:2207-2219

5. Aviv RI, Miszkiel K (2005) Orbital imaging: part 2. Intraorbital pathology. Clin Radiol 60:288-307

6. Bilaniuk LT (1999) Orbital vascular lesions: role of imaging. Radiol Clin North Am 37:169-183

7. Kapur R, Mafee MF, Lamba R, Edward DP (2005) Orbital schwannoma and neurofibroma: role of imaging. Neuroimaging Clin North Am 15:159-174

8. Ben Simon GJ, Annunziata CC, Fink J, Villablanca P, McCann JD, Goldberg RA (2005) Rethinking orbital imaging establishing guidelines for interpreting orbital imaging studies and evaluating their predictive value in patients with orbital tumors. Ophthalmology 112:2196-2207

9. Wenig B, Mafee MF, Ghosh L (1998) Fibro-osseous, osseous, and cartilaginous lesions of the orbit and paraorbital region. Correlative clinicopathologic and radiographic features, including the diagnostic role of CT and MR imaging. Radiol Clin North Am 36:1241-1259
10. Mafee MF, Haik BG (1987) Lacrimal gland and fossa lesions: role of computed tomography. Radiol Clin North Am 25:767-779

11. Mafee MF, Edward DP, Koeller KK, Dorodi S (1999) Lacrimal gland tumors and simulating lesions. Clinicopathologic and MR imaging features. Radiol Clin North Am 37:219-239

12. Azar-Kia B, Naheedy MH, Elias DA Mafee MF, Fine M (1987) Optic nerve tumours: the role of magnetic resonance imaging and computed tomography. Radiol Clin North Am 25:561-581

13. Hollander MD, Fitzpatrick M, O'Connor SG, Flanders AE, Tartaglino LM (1999) Optic gliomas. Radiol Clin North Am 37:59-71

14. Weber AL, Klufas R, Pless M (1996) Imaging evaluation of the optic nerve and visual pathway including cranial nerves affecting the visual pathway. Neuroimaging Clin North Am 6:143177

15. Wilms G, Raat H, Dom R, Thywissen C, Demaerel P, Dralands G et al (1995) Orbital cavernous haemangioma: findings on sequential Gd-enhanced MRI. J Comput Assist Tomogr 19:548-551

16. Forbes G (1996) Vascular lesions in the orbit. Neuroimaging Clin North Am 6:113-122

17. Tanaka A, Mihara F, Yoshiura T, Togao O, Kuwabara Y, Natori Y et al (2004) Differentiation of cavernous hemangioma from schwannoma of the orbit: a dynamic MRI study. AJR Am J Roentgenol 183:1799-1804

18. Eisen MD, Yousem DM, Loevner LA, Thaler ER, Bilker WB, Goldberg AN (2000) Preoperative imaging to predict orbital invasion by tumor. Head Neck $22: 456-462$

19. Schnall MD, Blume J, Bluemke DA, DeAngelis GA, DeBruhl N, Harms S et al (2006) Diagnostic architectural and dynamic features at breast MR imaging: multicenter study. Radiology 238:42-53
20. Yabuuchi H, Fukuya T, Hachitanda Y, Hachitanda Y, Tomita K, Koga M (2003) Salivary gland tumors: diagnostic value of gadolinium-enhanced dynamic MR imaging with histopathologic correlation. Radiology 226:345-354

21. van Rijswijk CS, Geirnaerdt MJ, Hogendoorn PC, Taminiau AH, van Coevorden $\mathrm{F}$, Zwinderman $\mathrm{AH}$ et al (2004) Soft-tissue tumors: value of static and dynamic gadopentetate dimeglumine-enhanced MR imaging in prediction of malignancy. Radiology 233:493-502

22. Reddick WE, Taylor JS, Fletcher BD (1999) Dynamic MR imaging of microcirculation in bone sarcoma. J Magn Reson Imaging 10:277-285

23. Shields JA, Shields CL, Scartozzi R (2004) Survey of 1264 patients with orbital tumors and simulating lesions. Ophthalmology 111:997-1008

24. Demirci H, Shields CL, Shields JA, Honavar SG, Mercado GJ, Tovilla JC (2002) Orbital tumors in the older adult population. Ophthalmology 109:243-248

25. Demirci H, Shields CL, Karatza EC, Shields JA (2008) Orbital lymphoproliferative tumors: analysis of clinical features and systemic involvement in 160 cases. Ophthalmology 115:1626-1631

26. Sullivan TJ, Valenzuela AA (2006) Imaging features of ocular adnexal lymphoproliferative disease. Eye 20:1189-1195

27. Polito E, Galieni P, Leccisotti A (1996) Clinical and radiological presentation of 95 orbital lymphoid tumors. Graefes Arch Clin Exp Ophthalmol 234:504-509

28. Shields JA, Shields CL, Epstein JA, Scartozzi R, Eagle RC Jr (2004) Primary epithelial malignancies of the lacrimal gland: the 2003 Ramon L font lecture. Ophthal Plast Reconstr Surg 20:10-21 